SANDIA REPORT SANDB3-05584i Unilinited Release

Printed July 1983

Ue-66e or $1677-2$ I. $1072 y$

\title{
Evaluation of Aqueous-Foam Surfactants for Geothermal Drilling Fluids
}

\section{SAND--83-0584}

DE83 016446

Peter B. Rand, Orelio J. Montoya

Prepared by

Sandia National Laboratories

Albuquerque, New Mexico 87185 and Livermore, Callfornia 94550

for the United States Department of Energy

under Contract DE-ACO4-76DPOO789
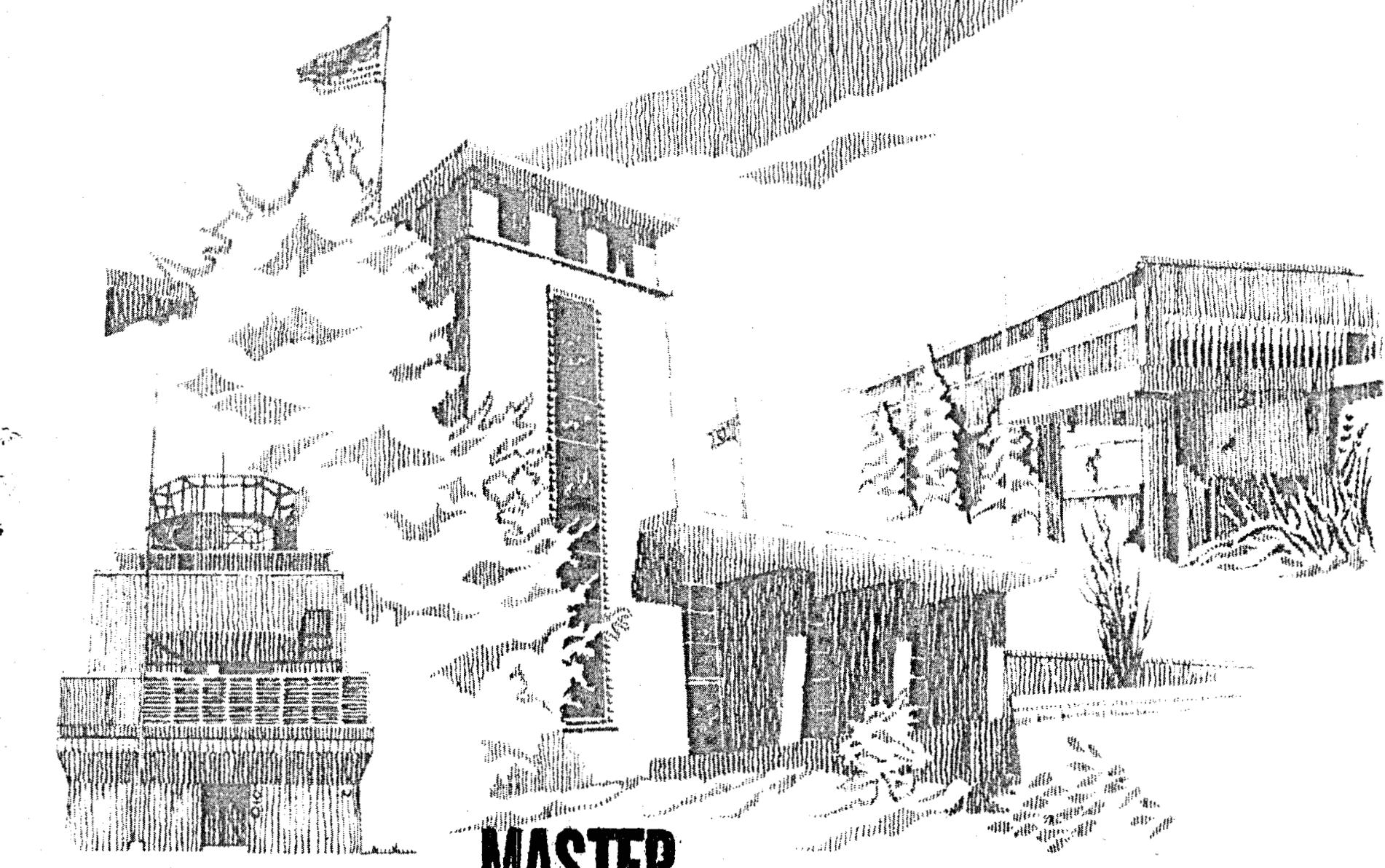

"Win $4 s_{n}$, WI

(4) 


\section{DISCLAIMER}

This report was prepared as an account of work sponsored by an agency of the United States Government. Neither the United States Government nor any agency Thereof, nor any of their employees, makes any warranty, express or implied, or assumes any legal liability or responsibility for the accuracy, completeness, or usefulness of any information, apparatus, product, or process disclosed, or represents that its use would not infringe privately owned rights. Reference herein to any specific commercial product, process, or service by trade name, trademark, manufacturer, or otherwise does not necessarily constitute or imply its endorsement, recommendation, or favoring by the United States Government or any agency thereof. The views and opinions of authors expressed herein do not necessarily state or reflect those of the United States Government or any agency thereof. 


\section{DISCLAIMER}

Portions of this document may be illegible in electronic image products. Images are produced from the best available original document. 


\title{
EVALUATION OF AQUEOUS-FOAM SURFACTANTS
}

FOR GEOTHERMAL DRILLING FLUIDS*

\author{
P. B. Rand \\ o. J. Montoya \\ Division 1813
}

Sandia National Laboratories

Albuquerque, New Mexico 87185**

\begin{abstract}
Aqueous foams are potentially useful ariliing and cleanout fluids for geothermal applications. Successful use of foams requires surfactants (foaming agents) that can survive in the hightemperature geothermal environment. In this study, solutions of aqueous-foam-forming surfactants have been exposed to $260^{\circ} \mathrm{C}$ $\left(500^{\circ} \mathrm{F}\right)$ and $310^{\circ} \mathrm{C}\left(590^{\circ} \mathrm{F}\right)$ in various chemical environments to determine if they can survive and make foams after exposure. Comparison of foams before and after exposure and the change in solution $\mathrm{pH}$ were used to evaluate their performance. Controlled liquid-volume-fraction foams, made in a packed-bed foam generator, were used for all tests. These tests have shown that many commercially available surfactants can survive short hightemperature cycles in mild acids, mild bases, and salt solutions as evidenced by their ability to make foams after exposure to high temperatures.
\end{abstract}

*This work performed at Sandia National Laboratories supporter by the U. S. Department of Energy under Contract DE-AC04-76-DP00789. 
TABLE OF CONTENTS

$\underline{\text { Page }}$

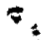

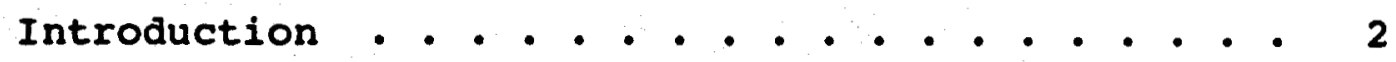

Experimental . . . . . . . . . . . . . 4

Results and Discussion . . . . . . . . . . 6

Conclusions . . . . . . . . . . . . . . 10

References . . . . . . . . . . . . . . 11 
Figures

Page

1. Aqueous Foam Generator . . . . . . . . . . . 25

2. Foam Drainage Test . . . . . . . . . . . 26

Tables

I Effect of $260^{\circ} \mathrm{C}$ - Deionized Water-Air Aging

Environment on Aqueous Foams and Solutions . . . 12

II Effect of $260^{\circ} \mathrm{C}$ - Deionized Water-Nitrogen Aging Environment on Aqueous Foams and Solutions . . . . 14

II Effect of $260^{\circ} \mathrm{C}-1.0 \mathrm{M}$ Sodium Chloride-Air Aging Environment on Aqueous Foams and Solutions . . . 15

IV Effect of $260^{\circ} \mathrm{C}-108$ Sodium Chloride 28 Calcium Chloride Brine-Air Aging Environment on Aqueous Foams and Solutions !. . . . . . . . . . . 16

$V$ Effect of $260^{\circ} \mathrm{C}-0.1 \mathrm{M}$ Hydrochloric Acid-Air Aging Environment on Aqueous Foams .......... . 17

VI Effect of $260^{\circ} \mathrm{C}-0.1 \mathrm{M}$ Sodium Hydroxide-Air Aging Environment on Aqueous Foams . . . . . . . . 18

VII Effect of $310^{\circ} \mathrm{C}$-Deionized Water-Air Aging Environment on Aqueous Foams and Solutions . . . . . . 19

VIII Effect of $310^{\circ} \mathrm{C}$-Deionized water-Nitrogen Aging Environment on Aqueous Foams and Solutions . . . 20

IX Effect of $310^{\circ} \mathrm{C}-\mathrm{Salt}$ Solutions-Air Aging Environments on Aqueous Foams and Solutionns . . . . . 21

$\mathrm{X}$ Effect of $310^{\circ} \mathrm{C}-\mathrm{Acidic}$ and Basic Solutions-Air Aging Environments on Aqueous Foams . . . . . . . . 22

XI Surfactant Performance Ratings After $260^{\circ} \mathrm{C}$ Aging in Various Chemical Environments ............ 23

XI Surfactant Performance Ratings After $310^{\circ} \mathrm{C}$ Aging in Various Chemical Environments . . . . . . . . 24 
INTRODUCTION

Aqueous foam is a potentially useful drilling fluid for underpressured geothermal reservoirs and workover fluid in many geothermal wells.1,2,3 Use of aqueous foams in these high temperature environments will require foaming agents or surfactants that are chemically stable and that will form stable foams at high temperatures. Some surfactants have been evaluated at high temperatures; 4,5 however, a broad survey has not been conaucted. Our interest in aqueous foams for geothermal drilling fluids led to an evaluation of more than fifty surfactants to determine their ability to withstand the combination of high temperatures and various chemical environments. Evaluation of foams made before and after the surfactant solution was exposed to high temperature was the primary tool used to evaluate their resistance to these environments. Although the test procedures and chemical environments developed for this study were selected to simulate the geothermal environment, the results are useful for other high temperature aqueous foam applications such as steam foam workover fluids for well cleanout and steam foam drives for oil recovery. 6

The geothermal arilling environment was simulated by exposing the surfactant solutions to a seven hour cycle with a two hour hold at the maximum temperature. Maximum temperatures of $260^{\circ} \mathrm{C}$ $\left(500^{\circ} \mathrm{F}\right)$ and $310^{\circ} \mathrm{C}\left(590^{\circ} \mathrm{F}\right)$ were used. Promising surfactants in preliminary tests were exposed to mild acids, mild bases, and salt solutions at high temperatures to simulate reservoir conditions, Foams, made with the surfactant solution before and after the solution was exposed to the high temperature cycle, 
were used to determine the effects of the high temperature exposure. The foams were made in a packed column foam generator which provided small celled foams with controlled liquid volume fractions. Liquid drainage from the foams and a visual evaluation of the cell structure were used to rate the foams.3 solution $\mathrm{pH}$ ร was also measured before and after high temperature exposure. These relatively simple tests allowed a large number of surfactants to be evaluated in various chemical environments before and after exposure to $260^{\circ} \mathrm{C}$ and $310^{\circ} \mathrm{C}$.

3 
Experimental

A wide variety of commercially available surfactants were evaluated Including amphoteric, anionic, cationic, and nonionic types. Most surfactants were evaluated as 0.5 weight percent active solutions. A few nonionic and other ethoxylated surfactants were evaluated as 1.0 weight percent active solutions because of their poor foaming performance at the 0.5 percent level. The surfactant solutions were contained in stainless steel mud aging vessels during the high temperature cycles. 0.25 liter of solution was placed in the 0.5 liter vessels for all tests. The seven hour temperature cycle used for all tests was as follows: a) ambient to test temperature, two hours, b) hold at test temperature, two hours and c) test temperature to ambient, three hours. All cycles were programmed with linear ramps up to temperature and down to ambient. Maximum temperatures of $260^{\circ} \mathrm{C}$ and $310^{\circ} \mathrm{C}$ were used. All fifty-six of the surfactants evaluated were tested after exposure to $260^{\circ} \mathrm{C}$ in deionized water. If successful in the environment, they were exposed to various chemical environments at $260^{\circ} \mathrm{C}$. The chemical environments included 1.0 molar sodium chloride, $10.0 \mathrm{wt} / 8$ sodium chloride $-2.0 \mathrm{wt} / \mathrm{q}$ calcium chloride brine, 0.1 molar hydrochloric acia, and 0.1 molar sodium hydroxide. Delonized water surfactant solutions were also evaluated with a nitrogen blanket to evaluate the effect of an oxygen free environment. Surfactants that proved successful In these tests were then evaluated at $310^{\circ} \mathrm{C}$ in the same environments. 
Foams for evaluation were made using a packed bed foam generator. The generator was made by filling a $10 \mathrm{~cm}$ section of a $12 \mathrm{~cm}$ long $46 \mathrm{~mm}$ I.D. stainless tube with a coarse stainless wool packed to a density of $1.0 \mathrm{gm} / \mathrm{cc}$. The solution and gas flow rates were controlled with fine metering valves fitted with micrometer heads. (Figure 1) This technique provided small-celled foams with controlled liquid volume fractions for evaluation. The liquid volume fraction was controlled to $0.045 \pm 0.05$ for all tests.

Foam drainage time, a measure of the short term or density stability of aqueous foams, was the primary quantitative test used to measure the effect of the high temperature cycles on the surfactant solutions. (Figure 2) The time to fifty percent arainage or weight loss was measured. The liquid volume fraction was determined for all foams evaluated by measuring the foam's weight and volume. The solution pH was measured before and after exposure with a Beckman Model $4500 \mathrm{pH}$ meter. All data presented are the average from three tests. A qualitative visual examination of the foam cell structure after exposure also proved to be an Important measure of the surfactant's usability. Ratings of good. fair, or poor cell structure were given. The ratings are defined as follows: good--small uniform cells; fair--nonuniform cell structure; poor-nonuniform cell structure with large cells. 
Results and Discussion

Organic surfactants exposed to either $260^{\circ} \mathrm{C}\left(500^{\circ} \mathrm{F}\right)$ or $310^{\circ} \mathrm{C}$ $\left(590^{\circ} \mathrm{F}\right)$ will degrade chemically. The objective of this study was not to measure chemical degradation but to determine the effect of the degradation on foams made with the surfactant solution after exposure to these high temperatures. At a given temperature exposure time is the most important factor. Our seven hour exposure cycle, with a two hour hold at the maximum temperature was selected to simulate the worst case for a foam drilling fluid in a geothermal well. If these surfactants were exposed for long times at these temperatures they would all fail. It is important to realize that only the surfactant solutions were exposed to the high temperature environment in this study. The foams were evaluated at room temperature.

One interesting result of ths study was the discovery that the high temperature exposure would in some cases improve the foam properties rather than degrade them. The surfactant degradation products in these cases were profoamers which would cause longer arainage times. As might be expected many surfactants would not make foam at 11 after exposure, elther because the surfactant was destroyed or the degradation products acted as antifoams. In a few cases we observed evidence of both pro and antifoamers from one surfactant. The evidence for this was provided by solutions that would not make fine uniform celled foams but would still give longer arainage times. This leads to an important point. As some degradation of the surfactant will occur at high temperatures the effect of the degradation products on the foams 
can be more important than the amount of surfactant degraded. For this reason surfactants with similar chemical stabilities can give very different results because of the effect of the degradation products on the foam.

The effects of exposing surfactant solutions to either $260^{\circ} \mathrm{C}$ or $310^{\circ} \mathrm{C}$ in various chemical environments are presented in Tables I-X. A summary of these data are presented in Tables XI and XII for $260^{\circ} \mathrm{C}$ and $310^{\circ} \mathrm{C}$ respectively using a performance rating system. The data on the surfactant includes the chemical type, trade name, and the weight-percent activity of the solution tested. The properties before exposure, liquid arainage time and solution pH are listed next. In Tables III-VI the percent change in arainage time caused by the various chemical environments is also given. These data compare the arainage time in deionized water to the drainage time in the specified environment before exposure. The effects of the high temperature exposure are shown by the percentage change in drain time, a cell structure rating, and the change in solution $\mathrm{pH}$.

A review of the data in Table I shows that more than half of the surfactants tested survived the $260^{\circ} \mathrm{C}$ exposure in the deionized water-air enviromment and were able to make good foams after exposure. Only small improvements were made by putting nitrogen over the surfactant solution--Table II. In the one molar sodium chloride solution, only one-third of the surfactants tested made good foams after the $260^{\circ} \mathrm{C}$ exposure--Table III. six of the surfactants would not make foams in the sodium chloride solution before the high temperature exposure. The toughest environment 
was the sodium chloride/calcium chloride brine as shown by the data in Table IV. Most of the anionic surfactants would not make foams because of the insolubility of their calcium salts. Only three surfactants made good foams after the $260^{\circ} \mathrm{C}$ exposure in this brine. The 0.1 molar hydrochloric acid environment also proved destructive to many surfactants as shown in Table v. Only six surfactants would make good foams after the $260^{\circ} \mathrm{C}$ exposure in the acid. The $260^{\circ} \mathrm{C}-0.1$ molar sodium hydroxide data, Table VII, reveal a number of surfactants that would make good foams after exposure. These data are important for foam drilling applications as a high $\mathrm{pH}$ is usually required for corrosion control. The effects of a $310^{\circ} \mathrm{C}$ exposure in the various chemical environments are presented in Tables VII-X. Only surfactants that showed promise in the $260^{\circ} \mathrm{C}$ tests were evaluated. As shown in Tables VII and VIII only two surfactants failed to make foam after exposure to $310^{\circ} \mathrm{C}$ in deionozed water. The adverse effect of dissolved salts is again shown by the data on surfactants exposed to $310^{\circ} \mathrm{C}$ in the sodium chloride and sodium chloride-calcium chloride brines-Table IX. Only three surfactants made good foams after exposure to the sodium chloride and only one in the calcium chloride-sodium chloride brine. The effects of the acidic and basic environments at $310^{\circ} \mathrm{C}$ are given in Table $\mathrm{x}$. Only one surfactant survived in the acid; however, six of nine made good foams after exposure in the 0.1 molar sodium hydroxide.

A summary of all testing is presented in Tables XI and XII for $260^{\circ} \mathrm{C}$ and $310^{\circ} \mathrm{C}$ using performance ratings. The ratings 
are defined as follows: E-excellent, less than 20.08 decrease in drainage time with good cell structure after exposure; G-good, more than 20.08 decrease in drainage time with good cell structure; P-poor, poor cell structure; U-unsatisfactory, no foam measuremints possible after exposure. The ratings show that several classes of surfactants may be suitable for high temperature applications. These include the alpha olefin sulfonates, quaternary aluminum chlorides, coco betaines, alkyl ethoxylates, alkyl aryl sulfonates, and several proprietary surfactants. Although no single surfactant was suitable for all environments, surfactants with excellent ratings in all environments tested at both $260^{\circ} \mathrm{C}$ and $310^{\circ} \mathrm{C}$ are available.

9 


\section{Conclusions}

These tests have shown that comercially available surfactants have sufficient chemical stability in high temperature aqueous environments to be useful for geothermal driliing and cleanout fluids. Because of their relatively low cost and good performance in these tests the alpha olefin sulfonates, as a class, appear to be the best surfactants available for these aplications. The alpha olefin sulfonates performed well in all environments except the sodium chloride/calcium chloride brine, and the $310^{\circ} \mathrm{C}, 0.1 \mathrm{M}$ hydrochloric acid. Other surfactants including the quarternary ammonium chlorids, coco betaines, alkyl aryl sulfonates, alkyl ethoxylates and several proprietary surfactants have also shown promise in these tests. Surfactant selection for a given application will depend on cost, anticipated chemical environment, compatibility with corrosion inhibitors, and the stability of aqueous foams made with the surfactant solution in high temperature environments.

As noted earlier in this report these high temperature exposure tests can only be used to evaluate the chemical stability or survivability of the surfactant in these aqueous environments. All foam evaluation was done at room temperature. Given surfactants with sufficient chemical stability the next step is to evaluate the structural stability of aqueous foams made with these surfactants at high temperatures and pressures in a suitable autoclave. These tests are under way. 
REFERENCES

1. S. 0. Hutchison, "Foam Workovers Cut Costs by 508," World. 011, November, 1969.

2. D. S. Pye, P. W. Fisher, "Foam Drilling and Workover in High Temperature We116." U. S. Patent 4,201,678, May 6, 1980 .

3. P. B. Rand, "Aqueous Foams for Geothermal Drilling Fluids: I. Surfactant Screening," Geothermal Resources Council

Transactions, volume 3, P. 565. September, 1978.

4. L. L. Handy, J. O. Amalfule, V. M. Ziegler, I. Ershacki "Thermal Stability of surfactants for Reservoir Application," Society of Petroleum Engineers Journal, October, 1982, P. 722 .

5. T. D. Elson, S. S. Marsden, Jr. "The Effectiveness of Foaming Agents at Elevated Temperatures Over Extended Periods of Time." Society of Petroleum Engineers Paper No. SPE-7116.

6. T. M. Doscher, E. C. Hammershaimb, "Field Demonstration of Steam Drive With Ancillary Materials," Journal of Petroleum Technology, July, 1982, P. 1535. 
TARIF. I

EFFECT OF $260^{\circ} \mathrm{C}$ - DFIONIZFD WATER - AIR AGTMG FNVIRONTHN ON AQUTOUS

FONMS AND SOLUTTONS

\begin{tabular}{|c|c|c|c|c|c|c|c|}
\hline CHEMICAL TYPE & TRADE NMF: & $\begin{array}{l}\text { Wr }-z \\
\text { ACIIVE }\end{array}$ & $\begin{array}{l}\text { ORIGI } \\
\text { DRATIAGF } \\
\text { TIME (MIN) }\end{array}$ & pH & $\begin{array}{l}\text { CHANGE IN } \\
\text { DRAIN (8) }\end{array}$ & $\begin{array}{l}\text { IIRR AGING } \\
\text { CFILL } \\
\text { STRUCTURE }\end{array}$ & $\begin{array}{l}\text { CHAKGE } \\
\text { IN pH }\end{array}$ \\
\hline ALPHA OLEFIN SULFONATE & SURFTOW S-398 & 0.5 & 9.13 & 11.1 & +14.9 & 0000 & +0.01 \\
\hline ALPHA OUEFIN SULPONAIE & SUIFOIFX AOS & 0.5 & 7.61 & 8.65 & +23.9 & 6000 & -4.30 \\
\hline ALPIA OLEFIN SULFONATE & IAKEWAY 301-10 & 0.5 & 8.44 & 8.05 & +9.1 & GOOD & -0.61 \\
\hline PROPRIEIARY ANIONIC & THERMOPHONM EKD & 0.5 & 7.32 & 10.3 & +21.3 & COOD & -1.42 \\
\hline ALKYL ARYL SULFONATE & SUNIFCK XIV & 0.5 & 7.26 & 6.45 & +19.3 & GOOD & -2.98 \\
\hline ANIONIC FLUOROCHEMICAI & FC-99 & 0.5 & 7.30 & 6.13 & +12.1 & G000 & -3.00 \\
\hline ALPEA OLEFIN SULFONAIE & SULFRAMIN 14/16 AOS & 0.5 & 7.45 & 6.70 & +8.6 & 0000 & -2.49 \\
\hline ALKYL ARYL SULFONAIF. & RICTONATE 60B & 0.5 & 8.06 & 5.88 & -0.6 & GOOD & -2.03 \\
\hline QUAT. ARMONIUM CHLORTDE & ADOGEN 461 & 0.5 & 7.66 & 7.23 & -0.3 & GOOD & -3.17 \\
\hline AMPHOTERIC FLUOROCHEMICAL & $1-4357$ & 0.5 & 11.30 & 9.49 & -34.6 & GOOD & -1.31 \\
\hline ALKYL ARYL SULFONATE & UDET-950 & 0.5 & 7.23 & 9.51 & -0.7 & GOOD & -2.48 \\
\hline QUAT. AMMONIUM CHLORIDE & ARQUAD $\$-50$ & 0.5 & 7.10 & 6.17 & -1.3 & GOOD & -1.50 \\
\hline COCO BETATNE & VEIVETEX BC & 0.5 & 6.68 & 5.03 & +3.3 & GOOD & +0.73 \\
\hline ANIONIC FLUOROCHEMICAL & $I-4587$ & 0.5 & 9.92 & 9.34 & -31.7 & GOOD & -6.68 \\
\hline EIHER SULFONATE & $80-69067$ & 0.5 & 6.22 & 5.24 & +4.3 & GOOD & -2.22 \\
\hline QUAT. AMMONIUM CHIORIDE & ARQUAD $C-50$ & 0.5 & 6.65 & 6.71 & -4.4 & GOOD & -1.64 \\
\hline PROPRIEIARY ANIONIC & $s-1100$ & 0.5 & 6.14 & 8.53 & +3.2 & GOOD & -4.41 \\
\hline ETHER SULFONAIE & $80-69068$ & 0.5 & 6.11 & 5.78 & -6.2 & $\mathbf{G O O D}$ & -2.88 \\
\hline SULFONATED QUAT. AMMONILM & EMS-121 & 0.5 & 5.34 & 6.85 & +5.6 & GOOD & -3.10 \\
\hline PROPRTEMARY & $M A-943$ & 0.5 & 8.29 & 7.24 & -32.6 & FATR & +1.69 \\
\hline AIKM ARYL SUIFONATE & NINEX N-300 & 0.5 & 10.20 & 6.76 & -45.3 & GOOD & -2.48 \\
\hline AIKML ARYL SUIFCNATE & PETRO BAF & 0.5 & 6.00 & 8.81 & $\cdot-13.2$ & $G 000$ & -4.01 \\
\hline PROPRIETARY NONIONIC & PEN-5E & 1.0 & 3.10 & 5.72 & +59.0 & GOOD & -0.27 \\
\hline ALKYL AMIDE SUIFONATE & IGFPON TC-42 & 0.5 & 5.33 & 7.64 & -8.4 & FAIR & -0.25 \\
\hline IMIDAZOITNE AMPHOTERIC & AMPHOTERGE: NEX & 0.5 & 8.23 & 7.22 & -41.3 & POOR & -0.50 \\
\hline ALKYL ARYL SUIFCNATE & PEIRO $\mathbf{P}$ & 0.5 & 4.92 & 9.77 & -9.3 & GOOD & -4.28 \\
\hline PROPRIETARY QUARIERNARY & M-QUAT 155-104 & 0.5 & 6.36 & 9.17 & -31.9 & FAIR & -2.49 \\
\hline
\end{tabular}




\begin{tabular}{|c|c|c|c|c|c|c|c|}
\hline \multirow{2}{*}{$\begin{array}{l}\text { TABLE I continued } \\
\text { CHEMTCAL TYPE }\end{array}$} & \multirow[b]{2}{*}{ TRADE NAME } & \multicolumn{3}{|c|}{ ORIGINAL } & \multicolumn{3}{|c|}{ AFTER AGINS } \\
\hline & & $\begin{array}{l}M=8 \\
\text { ACTIVE }\end{array}$ & $\begin{array}{l}\text { DRAINAGE } \\
\text { TIR (MTN) }\end{array}$ & nol & $\begin{array}{l}\text { CHANGF IN } \\
\text { DRATN (g) }\end{array}$ & CELL & $\begin{array}{l}\text { CHANGE } \\
\text { TN DoH }\end{array}$ \\
\hline PETROLETM SULFONATE & SUNIECK VII & 0.5 & 4.67 & 11.48 & -7.5 & GOOD & -0.07 \\
\hline ETHER SULFONATE & $79-69005$ & 0.5 & 4.43 & 3.64 & -3.2 & GOOD & -0.25 \\
\hline ETHOXMIATED QUAT. AMMONIUM & FIHOQUAD $18 / 25$ & 1.0 & 3.59 & 3.87 & +17.5 & FAIR & -0.03 \\
\hline ALKYL ARYL EIHER SULFONATE & TRTTON X-200 & 0.5 & 5.52 & 7.20 & -26.4 & GOOD & -2.21 \\
\hline ETHOXYLATED AMINE & MAZEAN C-15 & 0.5 & 2.63 & 9.70 & +53.6 & FAIR & -2.10 \\
\hline ALKYL ARYL ETHOXYLATE & WIIOONOL NP-120 & 1.0 & 3.44 & 6.52 & +10.7 & FAIR & -2.71 \\
\hline PROPRIEIARY ETHOXYIATED AICOHOT & MPD-5421 & 1.0 & 3.89 & 4.35 & -9.4 & GOOD & -0.72 \\
\hline PROPRIEIARY NONIONIC & MAZAMET 77 & 1.0 & 2.86 & 6.77 & +30.0 & GOOD & -3.22 \\
\hline PROPRIEIARY AMPHOTIRTC & $\mathbf{H C}-2$ & 0.5 & 6.17 & 5.29 & -41.6 & POOR & +3.33 \\
\hline PROPRIIEIARY ETHOXYIATED AICOATOL & MPD-5420 & 1.0 & 4.39 & 5.15 & -20.7 & GOOD & -0.33 \\
\hline EIHOXYLATED AMTNE & VARONIC K215 IC & 1.0 & 2.83 & 9.62 & +21.9 & FAIR & -2.85 \\
\hline PEIROLEIM SULFONAIE & PEIROSTEP 450 & 0.5 & 3.70 & 7.69 & -11.6 & POOR & -1.99 \\
\hline PEIROLEUM SULFONATE & PEIROSIEP 420 & 0.5 & 4.42 & 10.41 & -26.7 & FAIR & -6.12 \\
\hline PROPRIETARY ANIONIC & HONCO SUDS & 0.5 & 6.70 & 8.46 & -52.8 & FAIR & -5.99 \\
\hline ETHER SULFATE & TD-1 & 0.5 & 6.38 & 8.42 & -55.0 & FAIR & -6.02 \\
\hline PROPRIETARY & MAGCOFOAMER 77 & 0.5 & 5.34 & 6.64 & -47.5 & FAIR & -3.00 \\
\hline ALKYL EIHOXYLALE & WITCONOT SR-90 & 1.0 & 2.65 & 5.62 & +1.5 & GOOD & -2.05 \\
\hline ALKYL ETHOXYLATE & TRICOL IAI-23 & 1.0 & 3.18 & 6.58 & -19.2 & $\mathbf{6 0 0 0}$ & -2.78 \\
\hline PROPRIETARY & ADOFOAM BF-1 & 0.5 & 4.31 & 8.43 & -43.8 & GOOD & -5.91 \\
\hline PROPRIEIARY & $M A-945$ & 0.5 & 5.09 & 6.87 & -53.4 & POOR & -4.29 \\
\hline IMIDAZOLNNE AMPLOTERIC & MONATERIC CA-35 & 0.5 & 6.81 & 7.44 & - & OAM- & +1.12 \\
\hline PROPRIEIARY AMPHOTERIC & AMPEO B11-34 & 0.5 & 8.48 & 6.18 & 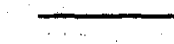 & OAM- & -1.07 \\
\hline AMPHOTERTC FLOROCHEMTCAL & ZONYL FSB & 0.5 & 12.40 & 4.18 & & OAM- & -0.02 \\
\hline IMIDAZOUTNE AMPHOTERIC & AMPHOTERGE K2 & 0.5 & 10.69 & 9.70 & & OAM- & -1.57 \\
\hline IMIDAZOLTNE AMPHOTERIC & MONATERIC 1000 & 0.5 & 5.01 & 8.92 & & OAM- & -1.88 \\
\hline ALKYL ESTER SULFONATE & IANTHANOL IAL & 0.5 & 6.43 & 6.35 & & OAM- & -2.39 \\
\hline ALKYL SULFONATE & AIXANOL, $189 \mathrm{~S}$ & 0.5 & 4.37 & 5.97 & $\longrightarrow$ & OAM- & -2.72 \\
\hline CATIONIC FLUOROCIIEYICAL & FC-135 & 0.5 & 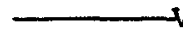 & . & & $x^{2}+4$ & \\
\hline ANIONIC FLUOROCHEMICAL & FC-95 & 0.5 & & MAK & $\pi$ & $\mathbb{E}$ FT & \\
\hline
\end{tabular}


EFFFCT OF $260^{\circ} \mathrm{C}$ - DEIONIZFD WATER -

NIIPOGEN AGING ENNIROMMFNT ON AOUROUS FOAMS AND SOUUTIONS

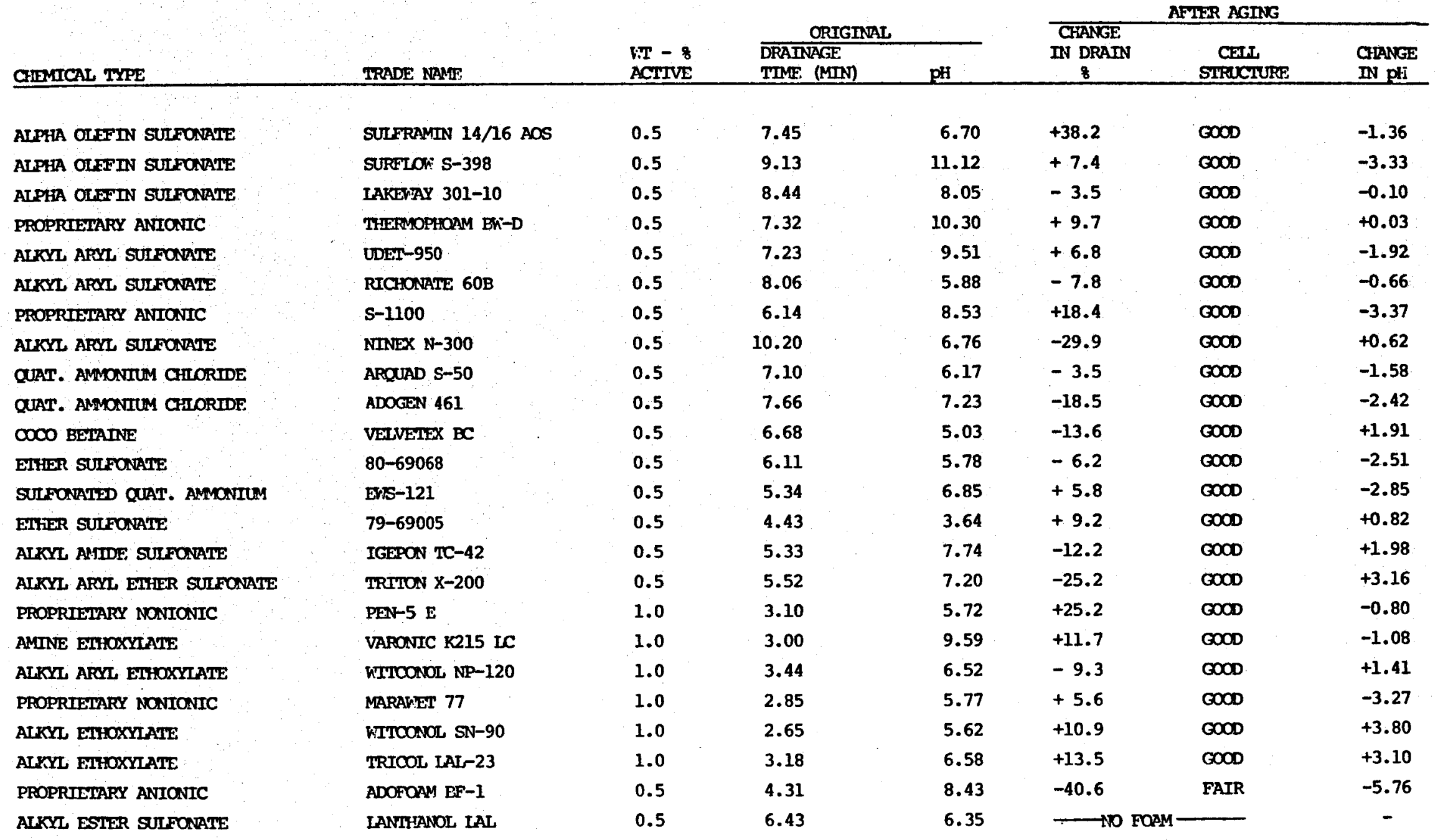


TABLE III

EFFECT OF $260^{\circ} \mathrm{C}$ - $1.0 \mathrm{M}$ SODTUM CHIORTDE -

AIR AGING ENITRONMENT ON AQUEOUS

FOAMS AND SOUUTIONS

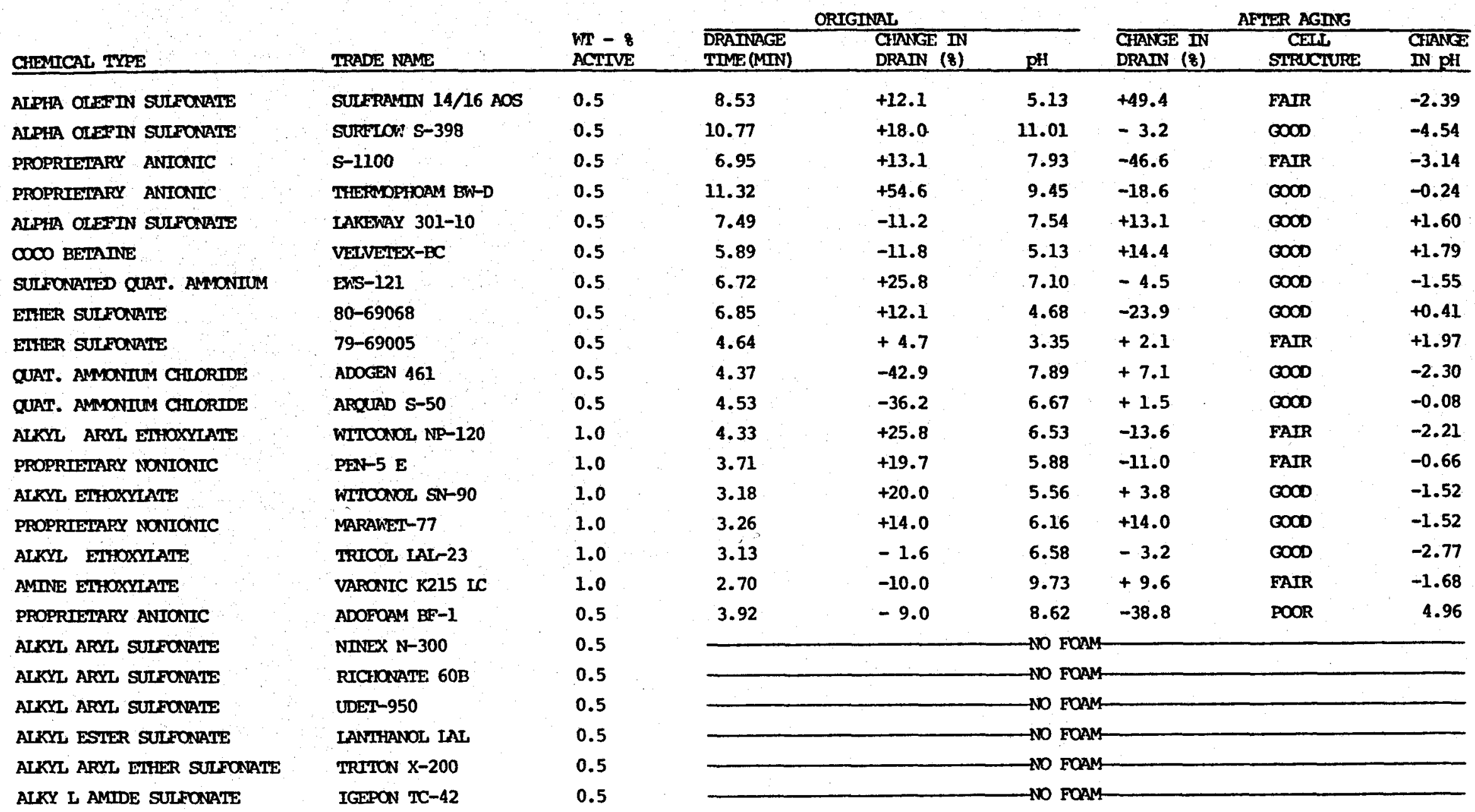


EFFFCT OF $260^{\circ} \mathrm{C}-10 \% / 2.08$ SODTUM CHIORIDF/CALCTIM CHLORTDF BRINE

- ATR AGING ENIROAMTI ON AQUBOUS FOAMS AND SOLUTIONS

\begin{tabular}{|c|c|c|c|c|c|c|c|c|}
\hline CHEMICAL TYPE & TRADE NAME & $\begin{array}{l}\text { WI - 8 } \\
\text { ACIIVE }\end{array}$ & $\begin{array}{l}\text { DRAINACAE } \\
\text { TMME (MIN) }\end{array}$ & $\begin{array}{l}\text { RIGINAL } \\
\text { CHANGE IN } \\
\text { DRMIN (8) }\end{array}$ & pII & $\begin{array}{l}\text { CHANGE IN } \\
\text { DRAIN (8) }\end{array}$ & $\begin{array}{l}\text { TER AGING } \\
\text { CETIL } \\
\text { SIRUCTURE }\end{array}$ & $\begin{array}{l}\text { CHANGes } \\
\text { IN pH }\end{array}$ \\
\hline OCO BEIATNE & VELVEIEX BC & 0.5 & 7.91 & +18.4 & 6.75 & -21.6 & FAIR & +0.04 \\
\hline QUAT. AMMONTUM CHIORIDE & ADOGEN 461 & 0.5 & 4.64 & -39.4 & 9.92 & +31.2 & GOOD & -4.09 \\
\hline SULFONATED QUAT. AMMONITI & ENS-121 & 0.5 & 6.89 & +29.0 & 7.67 & -13.9 & 6060 & -1.69 \\
\hline ETHER SULFONATE & $80-69068$ & 0.5 & 5.90 & -3.9 & 5.21 & -8.6 & GOOD & -0.60 \\
\hline EIIIER SUIFONAIE & $79-69005$ & 0.5 & 5.24 & +18.2 & 5.17 & -6.9 & FATR & -1.83 \\
\hline ALKYL ETHOXYAATE & TRTOOL LAL-23 & 1.0 & 3.37 & +6.0 & 6.04 & +40.6 & FAIR & -1.07 \\
\hline PROPRIETARY NONIONIC & MAXAWET 77 & 1.0 & 3.94 & +37.8 & 9.80 & +11.7 & POOR & -4.22 \\
\hline ALKYL EHTOXYIATE & IIIOCONOL SH-90 & 1.0 & 4.76 & +81.7 & 9.62 & -9.2 & FAIR & -4.58 \\
\hline AMINE EIHOXYLATE & VARONIC K215LC & 1.0 & 4.24 & +49.8 & 10.21 & -0.5 & POOR & -2.72 \\
\hline DROPRIEIARY ANIONIC & ADOFOAM BF-1 & 0.5 & 6.98 & +61.9 & 8.68 & -52.3 & POOR & -3.55 \\
\hline QUAT. AMMONIUM CHLORTDE & ARQUAD $S-50$ & 0.5 & 6.17 & -13.1 & 9.70 & $\longrightarrow$ N & - & -3.28 \\
\hline ALKYL ARYL EIHOXYLATE & VITLONOL NP-120 & 1.0 & & $\longrightarrow \sqrt{F}$ & OR FOAM & MFASUREMEN & OSSIBLE & \\
\hline PROPRIEIARY NONIONIC & PEN-5E & 1.0 & & $-\mathrm{v}$ & FOAM & MEASUREMPN & SSSIRLE- & \\
\hline ALPEA OLEFIN SUIFONATE & LAKEWAY 30I-10 & 0.5 & & & RFACLANI & IED OU & & \\
\hline ALPHA OLEFIN SULFONAIE & SULPRAMIN 14/16 AOS & 0.5 & & & RFACTANI & TFD OUT- & & \\
\hline ALPHA OLEFIN SULFONATE & SUPFLO S-398 & 0.5 & & & PFACTAM & IFD OTL & & \\
\hline PROPRIETARY ANIONIC & THFTMOPHOAM EN-D & 0.5 & & & RFACTAN & TED aT- & & \\
\hline PROPRIF:TARY ANIONIC & $s-1100$ & 0.5 & & & RFACTAN & TFD OUT & & \\
\hline ALKYL ARYL SULPONATE & UDFD-950 & 0.5 & & & RFACTAN & IFD OT- & & \\
\hline ALKYL ARYL SULFONATE & RICHOANATE $60 \mathrm{~B}$ & 0.5 & 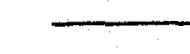 & & RFACTAN & IFD OUT- & & \\
\hline ALKYL ARYL SULFONAIE & NINEX N-800 & 0.5 & & & RFACTAN & IFD OUT- & & \\
\hline ALKYL ARYL ETHER SUIFONATE & TRIION $\mathrm{x}-200$ & 0.5 & & & FACTAN & MFD OUT- & & \\
\hline ALKYL AMIDE SULFONATE & IGEPON TC-42 & 0.5 & & & PACTAI & TED & & \\
\hline LKKY ESTER SULFONATE & IANMANOL LAL & 0.5 & & & CTMN & wis & & \\
\hline
\end{tabular}




\begin{tabular}{|c|c|c|c|c|c|c|}
\hline \multirow[b]{2}{*}{ CHENTCAL TYPE } & \multirow[b]{2}{*}{ TRADE NAME } & \multirow[b]{2}{*}{$\begin{array}{l}\text { WT }-8 \\
\text { ACTIVE }\end{array}$} & \multicolumn{2}{|c|}{ ORIGINAL } & \multicolumn{2}{|c|}{ AFIFR AGING } \\
\hline & & & $\begin{array}{l}\text { DRAINAGE } \\
\text { TIME (MIN) }\end{array}$ & $\begin{array}{l}\text { CHANGE IN } \\
\text { DRAIN (8) }\end{array}$ & $\begin{array}{l}\text { CFANGE IN } \\
\text { DRAIN (8) }\end{array}$ & CEEL STRLCTURE \\
\hline ALPHA OLEFIN SULFONATE & IAKTFAY 301-10 & 0.5 & 4.79 & -43.2 & +28.8 & COCD \\
\hline ALPHA OLEFTN SULFONATE & SUIFRAMIN $14 / 16$ AOS & 0.5 & 4.18 & -43.9 & +30.1 & GOOD \\
\hline QUAT. AMMONIUM CHIORIDE & ADOGEN-461 & 0.5 & 4.69 & -38.8 & +13.6 & COOD \\
\hline COCO BEIATNE & VEIVETEX BX & 0.5 & 5.32 & -20.4 & -13.3 & OOOD \\
\hline AMTNE EIHOXYATE & VARONIC K215LC & 1.0 & 3.03 & +1.0 & +32.0 & GOOD \\
\hline ALPHA OLEFIN SULFONATE & SURFLW S-398 & 0.5 & 4.34 & -52.5 & -8.3 & GOOD \\
\hline QUAT. AMONIUM CHIORIDE & ARQUAD S-50 & 0.5 & 5.78 & -18.6 & -35.4 & FAIR \\
\hline PROPRIETARY ANIONIC & THERMOPHOAM EN-D & 0.5 & 3.56 & -51.4 & -3.6 & HTGI DENSTIY \\
\hline PROPRIEIARY ANIONIC & $s-1100$ & 0.5 & 3.83 & -37.6 & -11.0 & FAIR \\
\hline AIKYL ARYL ETHOXYLATE & WITCONOL NP-120 & 1.0 & 3.40 & -1.2 & -9.4 & FAIR \\
\hline AIKYL EIHOXYIAIE & TRICOL IAI-23 & 1.0 & 2.93 & -7.9 & -11.3 & POOR \\
\hline EIHER SULFONATE & $79-69005$ & 0.5 & 5.24 & +18.2 & 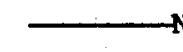 & t \\
\hline ALKYL ARYL SUIFONATE & UDEI-950 & 0.5 & 5.14 & -29.8 & -1 & 1 \\
\hline ETHER SULFONATE & $80-69068$ & 0.5 & 5.03 & -17.7 & -1 & $1-$ \\
\hline ALKYL ARYL SULFONATE & NTINEX N-300 & 0.5 & 4.32 & -57.6 & t & 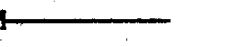 \\
\hline ALKYL AMIDE SULFONATE & IGEPON IC-42 & 0.5 & 3.92 & -26.5 & -1 & + \\
\hline ALKYL AFYL SULFONATE & RICHONAIE 60B & 0.5 & 3.91 & -51.5 & & \\
\hline PROPRIETARY NONIONIC & PEN-5E & 1.0 & 3.68 & +18.7 & & \\
\hline PROPRIETARY ANIONIC & ADOFOAM BF-1 & 0.5 & 3.58 & -16.9 & -1 & 4 \\
\hline ALKYL ARYL ETHER SULFONATE & TRITION $\mathrm{X}-200$ & 0.5 & 2.87 & -48.0 & & $y-$ \\
\hline PROPRIETARY NONIONIC & MAZANET 77 & 1.0 & 2.81 & -1.7 & & 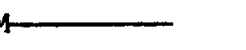 \\
\hline ALKYL EIHOXYLATE & HITCONOL SN-90 & 1.0 & 2.77 & +4.6 & & \\
\hline SULFONATED QUAT. AMMONIUM & EMS-121 & 0.5 & & & & \\
\hline ALKYL ESTER SULFONATE & IANIHANOL IAL & 0.5 & & & & \\
\hline
\end{tabular}


FFFPCT OF $260^{\circ} \mathrm{C}-0.1 \mathrm{M}$ SODTM FYDROXIDE - AIR AGING FNIPONMPT ON AOUEOUS FOAMS

\begin{tabular}{|c|c|c|c|c|c|c|}
\hline \multirow[b]{2}{*}{ CHEMTCAL TYPE } & \multirow[b]{2}{*}{ TRADE NAME } & \multirow[b]{2}{*}{$\begin{array}{l}\text { WT }-8 \\
\text { ACITVE } \\
\end{array}$} & \multicolumn{2}{|c|}{ ORIGINAL } & \multicolumn{2}{|c|}{ AFTER AGING } \\
\hline & & & $\begin{array}{l}\text { DRAIN } \\
\text { TIME (MIN) }\end{array}$ & $\begin{array}{l}\text { CHANGE IN } \\
\text { DRAIN (8) }\end{array}$ & $\begin{array}{l}\text { CAANGE IN } \\
\text { DRAIN (8) }\end{array}$ & CEIL STRUCTURE \\
\hline AIPHA OLEFIN SULFONATE & SULFRAMIN 14/16 AOS & 0.5 & 8.31 & +11.5 & +15.4 & GOOD \\
\hline ALPHA OIEFIN SULFONATE & SURFLOW S-398 & 0.5 & 8.45 & -7.5 & +0.3 & COOD \\
\hline AIKYL AMIDE SULFONATE & IGEPON $T C-42$ & 0.5 & 5.98 & +12.2 & +31.1 & GOOD \\
\hline ALPHA OLEFIN SULFONATE & IAKEWAY 301-10 & 0.5 & 7.99 & -5.3 & -7.4 & GOOD \\
\hline PROPRIEIARY ANIONIC & $s-1100$ & 0.5 & 5.70 & -7.2 & +25.3 & G000 \\
\hline ALKYL ARYL SULFONATE & UDET-950 & 0.5 & 6.26 & -13.4 & +8.1 & GOOD \\
\hline ALKYL ARYL SULFONAIE & NINEX N-300 & 0.5 & 5.67 & -44.4 & +4.9 & GOOD \\
\hline PROPRIEIAPY ANIONIC & THERNOPHOAM BW-D & 0.5 & 6.10 & -16.6 & -2.9 & GOOD \\
\hline AIKYL ARYL SULFONATE & RICHONATE $60 \mathrm{~B}$ & 0.5 & 5.25 & -34.9 & -5.9 & GOOD \\
\hline$\infty \infty \infty$ BETAINE & VELVETLXX BC & 0.5 & 3.94 & -41.0 & +21.8 & FAIR \\
\hline EHHER SUIFCNAME & $79-69005$ & 0.5 & 5.03 & +13.5 & -16.5 & FAIR \\
\hline ALKYL EIFOXYATE & WITCONOL SN-90 & 1.0 & 3.35 & +26.4 & -2.7 & GOOD \\
\hline PKOPRIEIARY NONIONIC & MAZAWEI-77 & 1.0 & 3.08 & +7.7 & +5.2 & 9000 \\
\hline ETHER SULFONATE & $80-69068$ & 0.5 & 6.09 & -0.3 & -51.4 & FAIR \\
\hline AMINE ETHOXYIATE & VARONIC $\mathrm{K}-215$ IC & 1.0 & 2.83 & -5.7 & 0.0 & GOOD \\
\hline ALKYL ARYL EIHOXYLATE & WITCONOL NP-120 & 1.0 & 3.21 & -6.7 & -11.8 & FAIR \\
\hline PROPRIEIARY NONIONIC & PEN-5E & 1.0 & 4.00 & +29.0 & -30.0 & GOOD \\
\hline ALKYL ETHOXYLATE & TRICOL IAI-23 & 1.0 & 2.79 & -12.3 & -1.8 & GOOD \\
\hline PROPRIETARY ANIONIC & ADOFOAM BF-1 & 0.5 & 4.17 & -3.2 & -35.0 & FAIR \\
\hline QUAT. AMMONIUM CHIORIDE & ARQUAD S-50 & 0.5 & 5.78 & -18.6 & -10 & \\
\hline SULFONATED QUAT. AMMONIUM & ENS-121 & 0.5 & 5.24 & -1.9 & $\rightarrow$ No & \\
\hline QUAT. AMMONILM CHLORIDE & ADOGEN 461 & 0.5 & 5.10 & -33.4 & $\longrightarrow$ NO & \\
\hline ALKYL ESTER SULFONATE & LANIFANOL IAL & 0.5 & 4.19 & -34.8 & $-N O$ & \\
\hline ALKYL ARYL ETHER SULFONATE & TRITION $X-200$ & 0.5 & 3.13 & -43.3 & $\longrightarrow$ NO & \\
\hline
\end{tabular}


TARUE VII

EFFECT OF $310^{\circ} \mathrm{C}$ - DETONIZFD MATFR - AIR AGING FWNIRONMFI

ON AOUTOUS FOAMS AND SOUUTIONS

\begin{tabular}{|c|c|c|c|c|c|c|c|}
\hline \multirow[b]{2}{*}{ CHEMICAL TYPE } & \multirow[b]{2}{*}{ TRADE NAME } & \multicolumn{3}{|c|}{ ORIGINAL } & \multicolumn{3}{|c|}{ AFIFR AGI } \\
\hline & & $\begin{array}{l}\text { hT - } 8 \\
\text { ACTIVE }\end{array}$ & $\begin{array}{l}\text { DRAINAGE. } \\
\text { TIME (MIN) }\end{array}$ & $\mathrm{pH}$ & $\begin{array}{l}\text { CHANGE IN } \\
\text { DRAIN (8) }\end{array}$ & $\begin{array}{l}\text { CELL } \\
\text { STRUCIURE }\end{array}$ & $\begin{array}{l}\text { CFANGE } \\
\text { IN PH }\end{array}$ \\
\hline CUAT. AMMONTUM CHLORIDE & ADOGEN 461 & 0.5 & 7.66 & 7.23 & +19.8 & $C 000$ & -1.47 \\
\hline ALPHA OLEFTN SULFONATE & SURFLO' S-398 & 0.5 & 9.13 & 11.12 & -7.0 & GOOD & -5.56 \\
\hline ALKM APCL SULPONATE & SUNIECK XIV & 0.5 & 7.26 & 6.95 & +12.7 & COOD & -3.05 \\
\hline PROPRIETARY ANIONIC & $s-1100$ & 0.5 & 6.14 & 8.53 & +32.1 & COOD & -4.48 \\
\hline ALKYL APOL SULFCNATE & UDET-950 & 0.5 & 7.23 & 9.51 & +1.8 & GOOD & -6.21 \\
\hline ALPHA OLEFIN SULFONATE & SUIFOIEX AOS & 0.5 & 7.61 & 8.65 & -5.5 & GOOD & -5.34 \\
\hline ANIONIC FLUOROCHIMICAL & FC-99 & 0.5 & 7.30 & 6.13 & -4.4 & 6000 & -2.03 \\
\hline ANIONIC FLOOROCHEMICAL & L-4587 & 0.5 & 9.92 & 4.34 & -32.0 & GOOD & -6.49 \\
\hline ALKYL ARYL SUIFONATE & PICHONATE 60B & 0.5 & 8.06 & 5.88 & -19.3 & GOOD & -2.48 \\
\hline EIHER SULFOAATE & $80-69067$ & 0.5 & 6.22 & 5.24 & +2.6 & GOOD & -2.74 \\
\hline EIHER SULFONATE & $80-69068$ & 0.5 & 6.11 & 5.78 & +4.1 & GOOD & -2.74 \\
\hline PROPRIEIARY ANIONIC & THEFMOPHOAM BW-D & 0.5 & 7.32 & 10.30 & -10.5 & GOOD & -1.23 \\
\hline ALPHA OLEFT SULFONATE & SULFRAMIN $14 / 16$ AOS & 0.5 & 7.95 & 6.70 & -24.6 & 0000 & -0.75 \\
\hline ALKYL ARYL SUIFONAIE & PETRO BAF & 0.5 & 6.00 & 8.81 & -9.3 & COOD & -1.35 \\
\hline ETFERR SUIFONATE & $79-69005$ & 0.5 & 4.43 & 3.64 & +15.3 & GOOD & +0.98 \\
\hline ALPHA OLEFIN SUIFONATE & LAKENAY 301-10 & 0.5 & 8.44 & 8.05 & -40.9 & GOOD & -3.10 \\
\hline SULFONATED QUAT. AMMONIUM & EMS-121 & 0.5 & 5.34 & 6.85 & -22.3 & HIGH DENSTTY & -1.80 \\
\hline ALKYL ARYL SULFONAIE & FEIRO $P$ & 0.5 & 4.92 & 9.77 & -18.1 & FAIR & -5.09 \\
\hline PROPRIEIARY NONIONIC & PEN-5 E & 1.0 & 3.10 & 5.72 & +29.5 & GOOD & -0.71 \\
\hline PROPRIEIARY NONIONIC & MAZAWET 77 & 1.0 & 2.86 & 6.77 & +33.5 & GOOD & -2.74 \\
\hline EITHOXYLATED AICOHOL & WITCONOL, SN-90 & 1.0 & 2.65 & 5.62 & +30.2 & GOOD & +0.62 \\
\hline ELFOXYLATED ALCOHOL & TRICOL IAL-23 & 1.0 & 3.18 & 6.58 & -3.1 & FAIR & -2.35 \\
\hline COOO BETAINE & VEIVETEX EX & 0.5 & 6.68 & 5.03 & - & $M-$ & +2.27 \\
\hline AMPHOIERTC FLUOROCHEMICAL & I 43357 & 0.5 & 11.30 & 9.49 & - & & -5.05 \\
\hline
\end{tabular}




\section{TABLE VIII}

EFFECT OF $310^{\circ} \mathrm{C}$ - DETONIZFD WATER - NITROGTN AGING FNIPONMFNT ON AQUEOUS FOAMS AND SOLUTIONS

\begin{tabular}{|c|c|c|c|c|c|c|c|}
\hline \multirow[b]{2}{*}{ CHEMICAL TYPE } & \multirow[b]{2}{*}{ TRADE NAME } & \multicolumn{3}{|c|}{ ORTGINAL } & \multicolumn{3}{|c|}{ AFITR AGING } \\
\hline & & $\begin{array}{l}\text { WT-8 } \\
\text { ACITVE }\end{array}$ & $\begin{array}{l}\text { DFAINAGE } \\
\text { TIPE (MIN) }\end{array}$ & pII & $\begin{array}{l}\text { CHANGE IN } \\
\text { DRAIN (8) }\end{array}$ & $\begin{array}{c}\text { CFIL } \\
\text { STRUCTURE }\end{array}$ & $\begin{array}{l}\text { CHAIIGE } \\
\text { IN PH }\end{array}$ \\
\hline PROPRIETARY ANIONIC & THEPMOPHOAM ENA-D & 0.5 & 7.32 & 10.30 & +35.2 & GOOD & -1.10 \\
\hline PROPRIEIARY ANIONIC & s-1100 & 0.5 & 6.14 & 8.53 & +41.4 & COOD & -3.92 \\
\hline ALPIA OTEFIN SUIFONATE & IAKEWAY $301-10$ & 0.5 & 8.44 & 8.05 & +2.1 & GOOD & -1.77 \\
\hline AIKYL ARYL SUIFONALE & UDET-950 & 0.5 & 7.23 & 9.51 & -5.0 & $\mathbf{6 0 0 0}$ & -5.16 \\
\hline QUAT. AMMONIUM CHLORIDE & ADOGEN 461 & 0.5 & 7.66 & 7.23 & -20.4 & GOOD & -1.62 \\
\hline AIPHA OLFFIN SUIFONATE & SURFLOW S-398 & 0.5 & 9.13 & 11.12 & -33.9 & 0000 & -5.20 \\
\hline ALPHA OLEFIN SULFONATE & SUIFRAMIN 14/16 AOS & 0.5 & 7.45 & 6.70 & -23.7 & $\mathbf{G O O D}$ & -2.89 \\
\hline ETHOXYIATED ALCOHOL & WITCONOL SN-90 & 1.0 & 2.65 & 5.62 & +98.1 & GOOD & +0.57 \\
\hline ETHER SULFONATE & $80-69068$ & 0.5 & 6.11 & 5.78 & +14.2 & COOD & -2.52 \\
\hline
\end{tabular}


TARIF, IX

EFFECT OF $310^{\circ} \mathrm{C}$ - SALT SOUUTIONS - AIR AGING FNIROMMFNT

ON AQUEOUS FOAMS AND SOLUTIONS

\begin{tabular}{|c|c|c|c|c|c|c|}
\hline & & & & & AFIER AGING & \\
\hline CHEMICAL TYPE & IRADE NAME & $\begin{array}{l}\text { DRAINAGE } \\
\text { TIME (MIN) }\end{array}$ & pHI & $\begin{array}{l}\text { CHANGE IN } \\
\text { DRAIN (8) }\end{array}$ & CELL STRUCIURE & $\begin{array}{l}\text { CHANGE } \\
\text { IN pH }\end{array}$ \\
\hline & & & SODIU & RWE & & \\
\hline AIPHA OLEFTN SUIFONATE & SURFLOW S-398 & 10.77 & 11.01 & +32.2 & FAIR & -3.99 \\
\hline ALPHA OLEFIN SULFONATE & LAKEMAY 301-10 & 7.49 & 7.54 & +84.6 & GOOD & -1.78 \\
\hline PROPRIEIAPY ANIONIC & $s-1100$ & 6.95 & 7.93 & +93.8 & FAIR & -2.25 \\
\hline PROPRIEIARY ANIONIC & THERMOPHOAM EN-D & 11.32 & 9.45 & -15.7 & HIG DFNSITY & -1.10 \\
\hline EIFER SULFOAATE & $80-69068$ & 6.85 & 4.68 & -2.2 & GOOD & -0.50 \\
\hline ALPHA OLEFIN SUIFONATE & SULFRAMTN 14/16 AOS & 8.53 & 6.91 & -40.6 & POOR & -1.56 \\
\hline QUAT. AMMONIUM CHLRIDE & ADOGEN 461 & 4.37 & 7.89 & +5.7 & GOOD & -1.91 \\
\hline ETHOXYLATED ALCOHOL & WITCONOL SN-90 & 3.18 & 5.56 & & A & - \\
\hline
\end{tabular}

IN 108 SODIUM CFIORTDF / 2.08CALCTUM CHLORIDE BRTNE

OUAT. AMMONIUM CHLORIDE ETHOXYLATED ALCOHOL EIHER SULFONATE
4.64
4.76
5.90

ADOGEN 461

WTICONOL SN-90

80-69068
9.92
8.06

$+23.7$

5.21

$+3.1$
GOOD

$-0.91$

POOR
$-2.71$

$-0.37$ 
TABIF, X

FFFECT OF $310^{\circ} \mathrm{C}$ - ACIDIC AND BASIC SOLUTIONS - AIR AGING ENVIRONMENIS

ON AQUEOUS FOAMS

\begin{tabular}{|c|c|c|c|c|}
\hline \multirow[b]{2}{*}{ CHE } & \multirow[b]{2}{*}{ TTADF NAMT } & ORIGINAL & \multicolumn{2}{|c|}{ AFTFR AGING } \\
\hline & & $\begin{array}{l}\text { DRATNAGE: } \\
\text { TIMF: (MMN) }\end{array}$ & $\begin{array}{l}\text { CHANGE IN } \\
\text { DRATN (8) }\end{array}$ & CEHL STRUCTURE \\
\hline
\end{tabular}

QUAT. AMMONIUM CHLORIDE ALPHA OLEFIN SUTFONATE PROPRIEIARY ANIONIC ALPYA OLEFIN SUIFONATE ETHER SUIFONATE ALPHA OUEFTN SULFONATE PROPRIETARY ANIONIC ETHOXYIATED ALCOHOS

PROPRIEIARY ANIONIC PROPRIETARY ANIONIC ALPHA OLEFIN SULPONATE ALPHA OLEFIN SULFONATE ALPHA OLEFIN SULPONATE ALKYL ARYL SULFONATE ETHER SUTFONATE ETFOXYIATED ALCOHOL

QUAT. AMONIUM CHIORTDE

\section{IN $0.1 \mathrm{M}$ HYDROCHLORIC ACTD} ALKYL ARXL SULFOAATE

\begin{tabular}{ll}
\hline ADOGEN 461 & 4.69 \\
SULFRAMIN 14/16 AOS & 4.18 \\
THERMOPHOAM BN-D & 3.56 \\
IAKEMAY 301-10 & 4.79 \\
80-69068 & 5.90 \\
UDET-950 & 5.14 \\
SURFIOW S-398 & 4.34 \\
S-1100 & 3.83 \\
WTIOONOL SN-90 & 2.77
\end{tabular}

\begin{tabular}{ll}
+6.8 & COOD \\
+2.6 & FAIR \\
+2.2 & FAIR \\
-39.4 & FATR \\
\hline & \\
\hline & \\
\hline & \\
\hline & \\
\hline & \\
\hline & \\
\hline
\end{tabular}

\section{IN $0.1 \mathrm{M}$ SODIUM HYDROXIDE}

$\begin{array}{llll}\text { THERMOPHOAM EN-D } & 6.10 & +18.8 & \text { COOD } \\ \text { S-1100 } & 5.10 & +26.0 & \text { GOOD } \\ \text { LAKEMAY 301-10 } & 7.99 & -11.6 & \text { COOD } \\ \text { SULFRAMIN 14/16 AOS } & 8.31 & -15.3 & \text { COOD } \\ \text { SURFLW S-398 } & 8.45 & -26.1 & \text { GOOD } \\ \text { UDET-950 } & 6.26 & -8.3 & \text { COOD } \\ \text { 80-69068 } & 6.09 & -47.3 & \text { FAIR } \\ \text { WITCONOL SN-90 } & 3.35 & -5.7 & \text { FAIR } \\ \text { ADOGEN } 461 & 5.10 & - & \end{array}$

4.69

3.56

4.79

5.14

4.34

2.77

5.10

\begin{abstract}
ADOCEN 461
\end{abstract}

$\therefore$

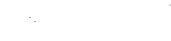

.
DRAIN (8)

CEIL SIRUCTURE 
TABTE XI

SURFACTANT PERPORMANCE RATINGS AFTER $260^{\circ} \mathrm{C}$ AGTMG IN VARIOUS CHEMICAL FNVIROMMFNTS

\begin{tabular}{|c|c|c|c|c|c|c|c|c|}
\hline CHEMICAL TYPE & TRADE NAME & $\begin{array}{l}\text { m }-8 \\
\text { ACIIVE }\end{array}$ & $\begin{array}{c}\text { WATER } \\
\text { AIR }\end{array}$ & $\begin{array}{c}\text { WATER } \\
\text { NITIBOGFN }\end{array}$ & $\begin{array}{l}1.0 \mathrm{M} \\
\mathrm{NaCl}\end{array}$ & $\begin{array}{r}10 / 2 \\
\text { BRINF. } \\
\end{array}$ & $\begin{array}{l}0.1 \mathrm{M} \\
\mathrm{HCl}\end{array}$ & $\begin{array}{l}0.1 \mathrm{M} \\
\mathrm{NaOH}\end{array}$ \\
\hline ALPHA OLEFIN SULPONATE & IAKEWAY $301-10$ & 0.5 & $\mathbf{E}$ & $\mathbf{E}$ & E & $\mathbf{v}$ & $\mathbf{E}$ & $\mathbf{E}$ \\
\hline QUAT. AMMONTUM CHLORIDE & ADOGEN 461 & 0.5 & $\mathbf{E}$ & $\mathbf{E}$ & $\mathbf{E}$ & $\mathbf{E}$ & $\mathbf{E}$ & $\mathbf{U}$ \\
\hline ALPHA OLEFIN SULFONATE & SURFLOW S-398 & 0.5 & $\mathbf{E}$ & E & $\mathbf{F}$ & $\mathbf{U}$ & $\mathbf{E}$ & $\mathbf{E}$ \\
\hline AIPHA OLEFIN SULFONATE & SULFRAMIN 14/16 AOS & 0.5 & $\mathbf{F}$ & $\mathbf{E}$ & G & $\mathbf{U}$ & $\mathbf{E}$ & $\mathbf{E}$ \\
\hline COOO BEIATNE & VELVELEX BC & 0.5 & $\mathbf{E}$ & F & $\mathbf{E}$ & $\mathbf{P}$ & $\mathbf{E}$ & $\mathbf{P}$ \\
\hline PROPRIETARY ANIONIC & THETMOPHOAM BN-D & 0.5 & $\mathbf{E}$ & $\mathbf{E}$ & $\mathbf{F}$ & $\mathbf{U}$ & $\mathbf{P}$ & $\mathbf{E}$ \\
\hline AIXYL ELTOXYLAIE & WTTCONOL SN-90 & 0.5 & $\mathbf{F}$ & $\mathbf{E}$ & $\mathbf{F}$ & $\mathbf{P}$ & $\mathbf{u}$ & $\mathbf{E}$ \\
\hline SULFONATED QUAT. AMMONIUM & ENS-121 & 0.5 & $\mathbf{E}$ & $\mathbf{E}$ & $\mathbf{E}$ & $\mathbf{E}$ & $\mathbf{u}$ & $\mathrm{U}$ \\
\hline EIHER SUIFONALE & $80-69068$ & 0.5 & $\mathbf{E}$ & F. & G & $\mathbf{E}$ & $\mathbf{U}$ & $\mathbf{P}$ \\
\hline PROPRIEIARY ANIONIC & $s-1100$ & 0.5 & $\mathbf{E}$ & $\mathbf{E}$ & $\mathbf{P}$ & $\mathbf{0}$ & $\mathbf{P}$ & $\mathbf{E}$ \\
\hline PROPRIEIARY NONIONIC & MAZAWET-77 & 1.0 & $\mathbf{E}$ & $\mathbf{E}$ & $\mathbf{P}$ & $\mathbf{P}$ & $\mathbf{U}$ & $\mathbf{E}$ \\
\hline QUAT. AMMONIUM CHIORIDE & ARQUAD $\$-50$ & 0.5 & $\mathbf{E}$ & $\mathbf{F}$ & $\mathbf{E}$ & $\mathbf{v}$ & $\mathbf{P}$ & $\mathbf{U}$ \\
\hline ALKYL ERIOXYIATE & TRICOL IAI-23 & 1.0 & $\mathbf{G}$ & $\mathbf{E}$ & E & $\mathbf{P}$ & $\mathbf{u}$ & $\mathbf{P}$ \\
\hline AMINE EITOXYIATE & VARONIC K215LC & 1.0 & $\mathbf{P}$ & $\mathbf{P}$ & $\mathbf{P}$ & $\mathbf{P}$ & E & $\mathbf{E}$ \\
\hline ALKYL ARYL SULFONATE & UDET-950 & 0.5 & $\mathbf{E}$ & $\mathbf{E}$ & $\mathbf{U}$ & $\mathbf{U}$ & $\mathbf{U}$ & $\mathbf{E}$ \\
\hline ALKYL ARYL SULFONATE & RICHONATE $60 \mathrm{~B}$ & 0.5 & $\mathbf{E}$ & $\mathbf{E}$ & U & $\mathbf{U}$ & $\mathbf{u}$ & $\mathbf{E}$ \\
\hline PROPRTEIARY NONIONIC & PEN-5E & 1.0 & $\mathbf{E}$ & $\mathbf{E}$ & $\mathbf{P}$ & $\mathbf{U}$ & $\mathbf{U}$ & G \\
\hline ETHER SULFONATE & $79-69005$ & 0.5 & $\mathbf{E}$ & $\mathbf{E}$ & $\mathbf{P}$ & $\mathbf{P}$ & $\mathrm{U}$ & $\mathbf{P}$ \\
\hline AIKYL ARYL SUIFONAIE & NINEX $N-300$ & 0.5 & G & $\mathbf{G}$ & $\mathrm{U}$ & $\mathrm{U}$ & U & $\mathbf{E}$ \\
\hline ALKYL ARYL ELHOXYLATE & WTICONOL NP-120 & 1.0 & $\mathbf{P}$ & $\mathbf{E}$ & $\mathbf{P}$ & $\mathrm{U}$ & $\mathbf{P}$ & $\mathbf{P}$ \\
\hline PROPRIEIARY ANIONIC & ADOFOAM BF-1 & 0.5 & G & $\mathbf{P}$ & $\mathbf{P}$ & $\mathbf{P}$ & $\mathbf{U}$ & $\mathbf{P}$ \\
\hline ALKYL AMIDE SULFONATE & IGIEPON TC-42 & 0.5 & $\mathbf{P}$ & $\mathbf{E}$ & U & $\mathrm{U}$ & $\mathbf{U}$ & $\mathbf{P}$ \\
\hline AIKYL ARYL EILER SULFONAIE & TRITON X-200 & 0.5 & G & $\mathbf{G}$ & $\mathbf{U}$ & $\mathbf{U}$ & $\mathbf{U}$ & $\mathbf{U}$ \\
\hline ALKYL ESTER SULFONATE & IANTHANOL LAL & 0.5 & $\mathbf{U}$ & $\mathbf{U}$ & $\mathbf{U}$ & $\mathrm{U}$ & U & $\mathrm{U}$ \\
\hline
\end{tabular}


TARIE XII

SURFACTANT PERFORMANCE RATTNGS AFTER $310^{\circ} \mathrm{C}$ AGTNG

IN VARTOUS CFIMIICAL ENWIRONMENIS

\begin{tabular}{|c|c|c|c|c|c|c|c|c|}
\hline CHEMICAL TYPE & TRADE NAME & $\begin{array}{l}\text { Wr - } 8 \\
\text { ACTIVE }\end{array}$ & $\begin{array}{c}\text { WAIFR } \\
\text { AIR }\end{array}$ & $\begin{array}{c}\text { WAIER } \\
\text { NIITOGEN }\end{array}$ & $\begin{array}{l}1.0 \mathrm{M} \\
\mathrm{NaCl}\end{array}$ & $\begin{array}{l}10 / 2 \\
\text { BRTNE }\end{array}$ & $\begin{array}{c}0.1 \mathrm{M} \\
\mathrm{HCl}\end{array}$ & $\begin{array}{l}0.1 \mathrm{M} \\
\mathrm{NaOH}\end{array}$ \\
\hline QUAT. AMMONIUM CHIORIDE & ADOGEN 461 & 0.5 & E & G & $\mathbf{E}$ & $\mathbf{E}$ & $\mathbf{E}$ & $\mathbf{u}$ \\
\hline ALPHA OLEFIN SULFONATE & IAKEMAY 301-10 & 0.5 & G & $\mathbf{E}$ & $\mathbf{E}$ & $\mathbf{u}$ & $\mathbf{p}$ & $\mathbf{E}$ \\
\hline PROPRIETARY ANIONIC & THEPMOPHOAM BPL-D & 0.5 & $\mathbf{E}$ & $\mathbf{F}$ & $\mathbf{P}$ & u & $\mathbf{P}$ & $\mathbf{E}$ \\
\hline PROPRIFIARY ANIONIC & $s-1100$ & 0.5 & $\mathbf{F}$ & $\mathbf{E}$ & $\mathbf{P}$ & $\mathbf{u}$ & u & $\mathbf{E}$ \\
\hline ALKYL ARYL SULFONAIE & UDET-950 & 0.5 & $\mathbf{E}$ & $\mathbf{E}$ & U & v & $\mathbf{u}$ & $\mathbf{E}$ \\
\hline FITHOXYLATED ALCOH:OL & PITCONOL SN-90 & 1.0 & $\mathbf{F}$. & $\mathbf{F}$ & $\mathbf{u}$ & $\mathbf{P}$ & $\mathbf{v}$ & $\mathbf{p}$ \\
\hline ALPHA OLEFIN SULPONATY & SUTFRAMIN $14 / 16$ AOS & 0.5 & G & G & $\mathbf{P}$ & $\mathbf{u}$ & $\mathbf{P}$ & $\mathbf{E}$ \\
\hline ALPHA OLEFIN SULFONATE & SURFLOW S-398 & 0.5 & $\mathbf{E}$ & G & $\mathbf{p}$ & U & $\mathbf{U}$ & G \\
\hline ETHFR SUIFONATE & $80-69068$ & 0.5 & $\mathbf{F}$ & $\mathbf{F}$ & u & u & $\mathrm{v}$ & $\mathbf{P}$ \\
\hline
\end{tabular}




\section{AQUEOUS FOAM GENERATOR}

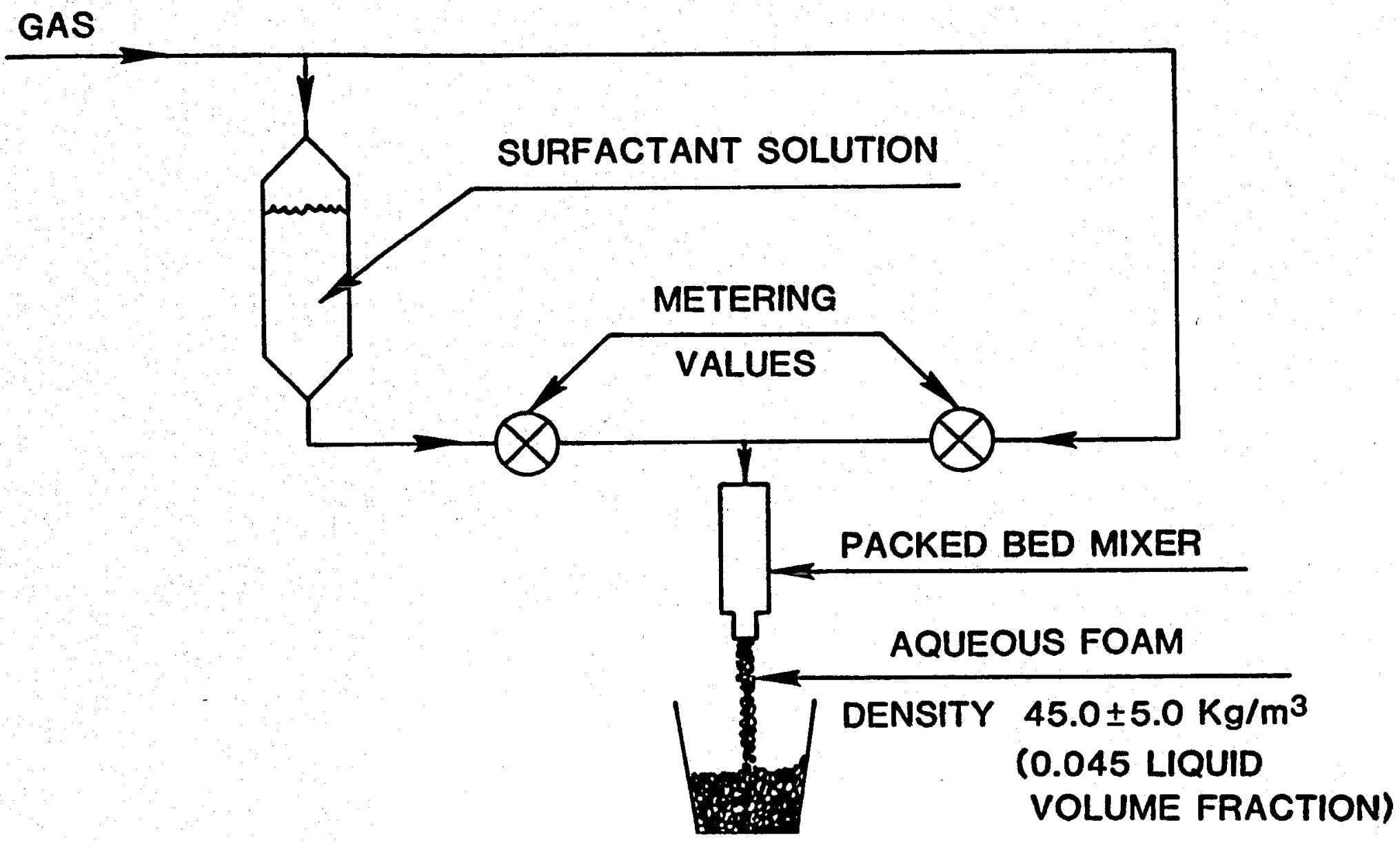

जั FIGURE 1 


\section{FOAM DRAINAGE TEST}

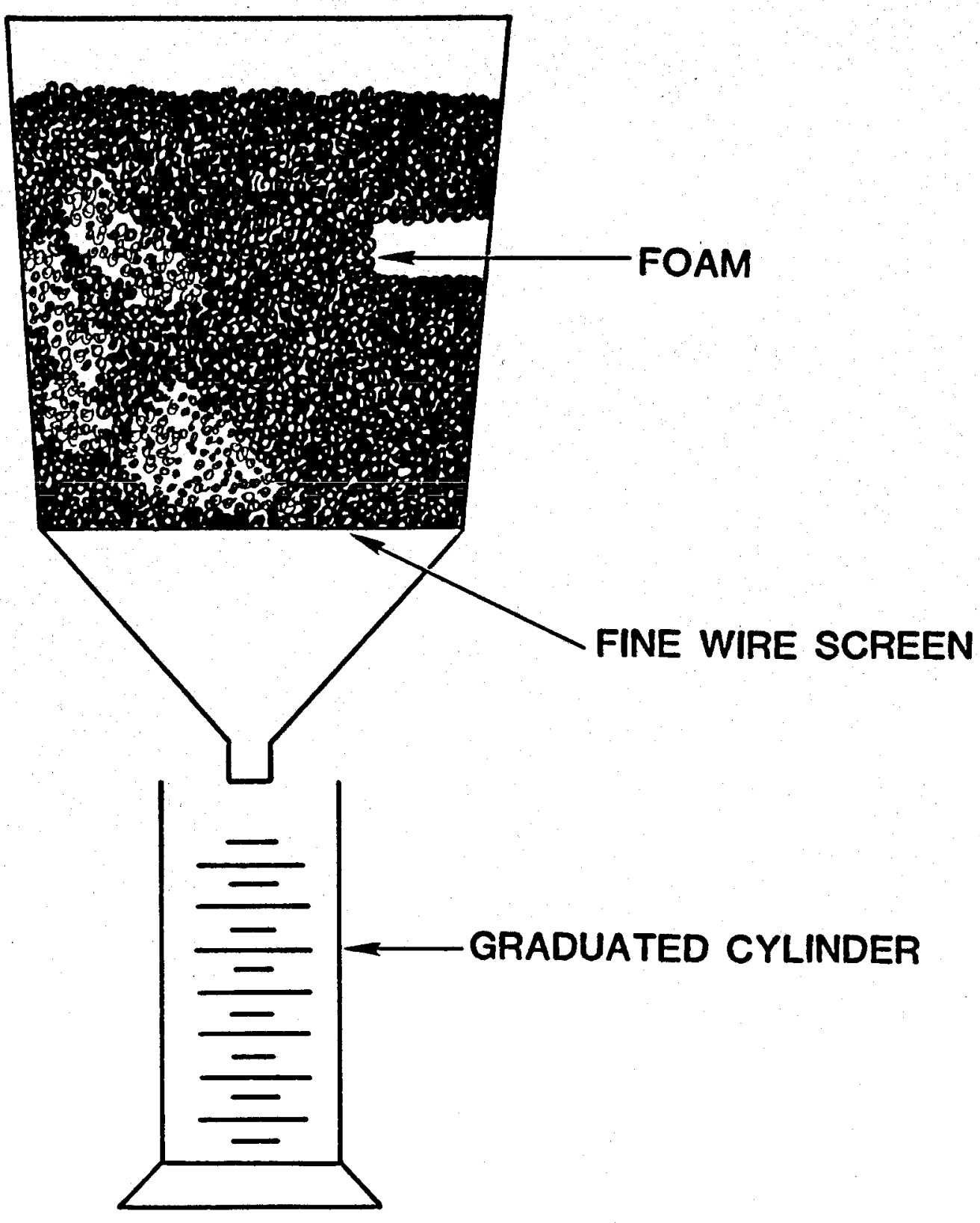


DISTRIBUTION: (UC66)

Tom Anderson

Venture Innovations

P. O. Box 35845

Houston, Texas 77035

Ed Bingman

Shell oil Company

Two Shell plaza

P. O. Box 2099

Houston. Texas 77001

Larry Diamond

Dyna-Drill

P. O. Box C-19576

Irvine, California 92713

Tom Turner

Philips Petroleum Company

Geothermal Operations

655 East 4500 South

Salt Lake City, Utah 84107

Jim Kingsolver

Geothermal Operations

Smith Tool

P. O. Box C-19511

Irvine, California 92713

John C. Rowley

Los Alamos National Labs

Mail stop 570

Los Alamos, New Mexico 87545

Ed Martin

Superior 011

Eastern Division

P. 0. Box 51108 ocs

Lafayette, Louisiana 70505

B. J. Livesay

129 Liverpool

Cardiff, California 92007

William R. Clemente

NL Baroid

P. O. Box 1675

Houston, Texas 77001
Gene Polk

NL Baroid

6400 Uptown Blvd. N.E., 365 W

Albuquerque, New Mexico 8711

James $w$. Langford

Security Division

Dresser Industries, Inc.

P. O. BoX 24647

Dallas, Texas 75224

John E. Fontenot

NL Petroleum Services

P. O. Box 60087

Houston, Texas 77205

Del E. Pyle

Union Geothermal Division

Union Oil Co. of California

Union oil Center

Los Angeles, California 90017

William D. Rumbaugh

Research \& Development

Otis

P. O. Box 34380

Dallas, Texas 75234

Dwight Smith

Halliburton

Drawer 1431

Duncan, Oklahoma 73533

Tom Warren

Amoco Production Company

Research Center

P. O. Box 591

Tulsa, Okalahoma 74102

H. E. Mallory

P. O. Box 54696

Tulsa, Oklahoma 74155

Myron T. Pike

$3 \mathrm{M}$

236-2A 3 M Center

st. Paul, MN 55144 
A. J. Havlik Associates 5540 Pine Cone Road

La Crescenta, CA 91214

G. W. Aderson

Chevron USA, Inc.

P. O. Box 5355

Bakersfield, CA 93108

Lewis Norman, Ph.D.

Halliburton Services

P. O. Box 1431

Duncan, OK 73533

J. H. Duerkson

Chevron OLl Field Research Co. P. O. Box 446

La Habra, CA 90631
Dr. Melvin Friedman

Professor of Geology

Center for Tectonophysics

and Dept. of Geology

Texas A \& M University

College Station, Texas 77843

U. S. Department of Energy (4)

Geothermal Hydropower

Technologies Division

Forrestal Bldg., CE 324

1000 Independence Ave.. S.W.

Washington, D.C. 20585

Attn: J. Bresee

D. Clements

R. Toms

D. Allen

W. P. Grace, DOE/ALO

Nuclear and Geosciences

Division

$\begin{array}{ll}3141 & \text { L. J. Erickson (5) } \\ 3151 & \text { W. L. Garner (3) } \\ 3154-3 & \text { C. H. Dalin (25) } \\ & \text { for DoE/TIC } \\ 6000 & \text { E. H. Beckner } \\ 6200 & \text { V. L. Dugan } \\ 6240 & \text { R. R. Traeger } \\ 6241 & \text { J. R. Kelsey (10) } \\ 6241 & \text { C. C. Carson } \\ 6246 & \text { B. Granoff } \\ 6247 & \text { P. J. Hommert } \\ 6300 & \text { R. W. Lynch } \\ 6330 & \text { W. D. Weart } \\ 8214 & \text { M. A. Pound }\end{array}$

